\title{
Correlação entre o índice tornozelo-braço antes e após teste de deslocamento bidirecional progressivo
}

\author{
Correlation between ankle-brachial index before and after \\ shuttle walk test
}

\author{
Inácio Teixeira da Cunha-Filho, Danielle Aparecida Gomes Pereira, André Maurício Borges de Carvalho, \\ Júlia Polcaro Garcia, Luciana Morais Mortimer, Inalda Cunha Burni*
}

\section{Resumo}

Contexto: A alteração de fluxo sangüíneo observada nos pacientes com doença arterial obstrutiva periférica (DAOP) contribui para a redução da capacidade deambulatórida. Entretanto, ainda existe uma grande variabilidade nas correlações entre medidas inferenciais de comprometimento de fluxo e testes de deslocamento.

Objetivo: Estabelecer o nível de correlação entre as medidas do índice tornozelo-braço (ITB), pré e pós-esforço, com um novo teste de deambulação chamado teste de deslocamento bidirecional progressivo (TDBP).

Métodos: Vinte e um pacientes claudicantes, com diagnóstico de DAOP, tiveram registrados o ITB antes e após a realização de um teste de caminhada no solo, com controle externo e progressivo de velocidade (TDBP).

Resultados: Foram registrados a distância (261,07 $\pm 160,63$ metros), o tempo $(292,30 \pm 122,61$ segundos) e a velocidade $(1,23 \pm 0,34 \mathrm{~m} / \mathrm{s})$ obtidos no início do surgimento de sintoma claudicante, bem como durante o surgimento de sintoma limitante $(369,52 \pm 157,97$ metros, $377,71 \pm 104,60$ segundos, $1,46 \pm 0,29 \mathrm{~m} / \mathrm{s}$, respectivamente). A média do ITB de repouso foi de $0,66 \pm 0,14$, e de pós-esforço foi de $0,42 \pm 0,19$. Não se observou nenhuma correlação importante entre as variáveis do teste (distância, tempo e velocidade) com o ITB de repouso e nem após esforço.

Conclusão: O tempo, velocidade e distância de surgimento de sintoma claudicante e de sintoma claudicante limitante durante o teste de caminhada progressiva são independentes da medida inferencial de fluxo sangüíneo através do ITB de repouso e pós-exercício.

Palavras-chave: Claudicação, caminhada, fluxo.

\section{Introdução}

A combinação de medidas de índice tornozelobraço (ITB) com testes de caminhada tem sido utilizada para melhor compreensão do grau de comprometimento do paciente com doença arterial obstrutiva periférica (DAOP), por associar medida inferencial de

\begin{abstract}
Background: Patients with peripheral occlusive artery disease (POAD) show changes in blood flow that may impair their walking ability. However, variability between inferential measurements of blood flow and walking performance is still high.
\end{abstract}

Objective: To correlate the ankle-brachial index (ABI) before and after performing the shuttle walk test (SWT).

Methods: Twenty-one patients with claudication due to POAD had their ABI values registered before and after walking based on a progressive external controlled speed walking protocol.

Results: Distance $(261.07 \pm 160.63 \mathrm{~m})$, time $(292.30 \pm 122.61$ seconds) and speed $(1.23 \pm 0.34 \mathrm{~m} / \mathrm{s})$ obtained at claudication onset and when the limiting walking symptom started $(369.52 \pm 157.97 \mathrm{~m}$, $377.71 \pm 104.60$ seconds, $1.46 \pm 0.29 \mathrm{~m} / \mathrm{s}$, respectively) were registered. Mean ABI before and after the SWT was $0.66 \pm 0.14$ and $0.42 \pm 0.19$, respectively. There was no substantial correlation between ABI preand post-walking and the variables obtained with the SWT (distance, time and speed).

Conclusion: Time, speed and distance for claudication onset and walking limiting symptom during a progressive speed walking protocol are independent of inferential blood flow measurements obtained by $\mathrm{ABI}$ before and after exercise.

Keywords: Claudication, walking test, flow.

integridade de fluxo sangüíneo com capacidade funcional ${ }^{1}$. Devido à vasodilatação e incapacidade de aumentar o fluxo depois do nível de obstrução aterosclerótica, o ITB medido após a atividade física (ITB-e) tende a cair em relação aos níveis de repouso. Assim, o ITB-e é considerado de melhor valor prognóstico que o ITB de

* Centro Universitário de Belo Horizonte (UNI-BH), Belo Horizonte, MG.

Projeto parcialmente financiado (bolsa auxílio pesquisa) pela FAPEMIG.

Artigo submetido em 13.06.07, aceito em 19.09.07.

J Vasc Bras. 2007;6(4):332-338.

Copyright $\odot 2007$ by Sociedade Brasileira de Angiologia e de Cirurgia Vascular 
repouso (ITB-r) ${ }^{2,3}$. Além disso, o ITB-e tem valor aditivo no diagnóstico da obstrução arterial periférica quando se trata de indivíduos cujo ITB-r é normal, mas apresenta queda importante com o esforço ${ }^{4}$. A redução da pressão sistólica após o exercício é considerada um dos indicadores mais sensitivos para detecção de estenose hemodinamicamente significativa ${ }^{5}$.

Entretanto, se por um lado o ITB-r é universalmente aceito como um indicador clínico do grau de comprometimento no fluxo sangüíneo, ele nem sempre apresenta correlações satisfatórias ou significativas com performance em testes funcionais ${ }^{6}$. O nível de correlação entre o ITB-r e distância caminhada até o início de claudicação $(r=0,17)$ e com claudicação máxima $(r=0,24)$, durante protocolo em esteira rolante com carga constante, é bastante baixo ${ }^{7}$. Contudo, quando o protocolo é de carga progressiva máxima, esses níveis sobem para 0,48 e 0,61 , respectivamente ${ }^{8,9}$.

O ITB-e pode, potencialmente, apresentar níveis de correlação com performance um pouco melhores, já que o portador de DAOP usualmente apresenta uma queda de ITB com o esforço. Entretanto, são necessárias mais investigações para se estabelecer a utilidade e viabilidade do ITB-e em testes de caminhada no solo.

$\mathrm{O}$ teste de esteira rolante (TR) fornece uma mensuração objetiva na performance da caminhada. Entretanto, é oneroso, muitas vezes requer pessoal especializado para utilização, nem sempre se encontra disponível em clínicas e não reproduz uma forma usual de deambulação. A confiabilidade teste-reteste, para tempo de surgimento de dor inicial e limitante, é baixa e bastante variável ${ }^{8,9}$. Naturalmente, essa variabilidade na estabilidade das medidas de distância observada com a esteira rolante reflete, em princípio, os múltiplos protocolos e/ou forma como os testes são conduzidos.

Testes de caminhada no solo têm sido propostos para avaliação funcional do paciente claudicante, tendo em vista a variabilidade dos resultados observados nos TR, falta de consenso entre a definição do melhor protocolo para esteira e as dificuldades técnicas e custo associados a este instrumento. $\mathrm{O}$ teste de caminhada de 6 minutos (T6M) foi desenvolvido como uma forma mais barata e alternativa para medir a capacidade funcional, e seu objetivo é medir a distância que o paciente é capaz de percorrer em um período de 6 minutos ${ }^{10}$. Contudo, o protocolo também apresenta problemas de padronização e maior suscetibilidade à variação, pois a velocidade do teste não é controlada e a performance pode ser influenciada pela motivação e encorajamento do paciente $^{10}$.

Um outro teste de caminhada de solo para avaliação de resistência aeróbica foi recentemente apresentado na literatura, denominado teste de deslocamento bidirecional progressivo (TDBP) ${ }^{11}$. Nesse teste, além do indivíduo poder desenvolver uma atividade de deambulação mais familiar, ou seja, caminhar no solo ao invés da esteira rolante, a velocidade é controlada externamente por um sinal sonoro previamente gravado. A velocidade aumenta a cada minuto e o teste só é interrompido quando o paciente não consegue acompanhar a velocidade previamente estabelecida para um estágio específico. É um teste de custo baixo e velocidade progressiva, oferecendo, portanto, maior possibilidade de revelar mais fielmente a capacidade funcional do paciente. Assim, o TDBP agrega valores presentes na esteira rolante (controle externo de velocidade) e no T6M (caminhada em superfície estável e mais representativa da deambulação diária). O TDBP também apresenta propriedades psicométricas superiores ao $\mathrm{T} 6 \mathrm{M}$ e ao $\mathrm{TR}^{10,12}$.

Tendo em vista que ainda não há consenso sobre qual ITB mais apropriado (repouso versus pós-esforço) para avaliação do grau de comprometimento do fluxo sangüíneo para membros inferiores, e que os protocolos de testes funcionais envolvendo a deambulação para a avaliação do paciente claudicante sejam variáveis, é necessário que aumentem as investigações acerca da associação das medidas inferenciais de fluxo com protocolos de performance mais funcionais.

Portanto, o objetivo deste trabalho é pesquisar o grau de associação do ITB pós-esforço com um teste de deambulação que se aproxime mais da atividade funcional e que apresente maior estabilidade em relação ao surgimento de sintomas.

\section{Métodos}

Indivíduos de ambos os sexos, independentes de etnia ou idade, portadores de DAOP comprovada clínica e/ou 
ultra-sonograficamente foram contatados para participar do estudo. Os pacientes faziam parte do programa de reabilitação vascular do Núcleo de Estudos de Doenças Vasculares Periféricas da Clínica Escola do Centro Universitário de Belo Horizonte (UNI-BH). O estudo teve início após ter sido aprovado pelo Comitê de Ética em Pesquisa com Seres Humanos do Hospital SOCOR e cada participante se juntou ao programa depois de assinar um termo de consentimento, contendo todas as informações inerentes ao estudo.

Foram incluídos no estudo pacientes adultos com DAOP que apresentaram ITB-r entre 0,9 e 0,4 , sem dor em repouso e com claudicação intermitente.

Foram excluídos pacientes que apresentassem ITB-r $>0,9$ ou $<0,4$, ou com dor em repouso. Pacientes não aptos a realizar exercício, tais como portadores de insuficiência cardíaca congestiva grave, angina instável, arritmias, que não cooperassem com a realização do teste; pacientes cujo ITB fosse inaudível e, conseqüentemente, não mensurável, e pacientes diabéticos, exceto os que apresentassem sintoma de dor, também foram excluídos do projeto.

\section{Procedimentos}

\section{Mensuração do ITB}

Para medida do ITB-r, o participante permaneceu em decúbito dorsal por um período de 5 minutos antes do início das medidas. Um manguito específico para membros superiores foi utilizado para avaliação das pressões sistólicas de ambos os membros. O manguito foi inflado a $20 \mathrm{mmHg}$ acima da pressão arterial sistólica (PAS). O registro da pressão foi feito com um aparelho Doppler portátil (MEDPEJ modelo DV-2001 Brasil), posicionado sobre a artéria braquial na fossa cubital.

Um manguito específico para membro inferior foi posicionado acima do maléolo e inflado a $20 \mathrm{mmHg}$ acima da PAS, para aferição das pressões sistólicas de cada membro inferior. A sonda do Doppler foi posicionada sobre as artérias tibial posterior e dorsal do pé. Embora o ITB seja usualmente obtido através da razão entre os valores mais altos das pressões sistólicas registradas no membro inferior e superior, este estudo optou por utilizar o valor mais baixo de pressão sistólica aferida no membro inferior, por julgar que a maior obstrução tenha maior impacto sobre o funcionamento do membro inferior ${ }^{2}$. O membro inferior que gerou o menor ITB foi utilizado como membro referência para registro do ITB após o teste de caminhada.

Após o teste de caminhada, o paciente foi posicionado imediatamente em decúbito dorsal e fez-se o registro da pressão do membro inferior e superior, nesta ordem. Porém, neste momento, apenas o membro previamente identificado com o mais baixo ITB foi avaliado.

\section{Teste de deslocamento bidirecional progressivo (TDBP)}

Nesse teste, o participante percorreu uma distância de 10 metros, delimitada por dois cones colocados no solo. O participante deu voltas consecutivas em torno de ambos os cones, com velocidades que aumentaram progressivamente até a exaustão, presença de sintoma claudicante ou incapacidade de manter o ritmo de deslocamento previamente estabelecido. A velocidade de deslocamento foi aumentada a cada minuto $(0,17 \mathrm{~m} / \mathrm{s}) \mathrm{e}$ controlada por sinais de áudio, gerados por aparelho de som portátil. O TDBP consistiu de 12 níveis com duração de 1 minuto cada, sendo que a velocidade inicial foi de $0,5 \mathrm{~m} / \mathrm{s}$ até o máximo de $2,37 \mathrm{~m} / \mathrm{s}^{11}$.

Durante o TDBP, o paciente fez uso de uma cinta sobre o tórax, contendo um transmissor portátil. Um cardiofrequencímetro da marca Polar ${ }^{\mathrm{TM}}$ (modelo Sport Tester - EUA) captou os sinais emitidos pelo transmissor afixado ao tórax, a fim de registrar a freqüência cardíaca (FC) durante todo o teste de caminhada. Ao final de cada minuto, a FC foi anotada.

As variáveis de interesse com esse teste foram distância, tempo e velocidade em que a dor claudicante iniciou e em que o sintoma limitante fez interromper o teste.

\section{Análise estatística}

Para a estatística descritiva, os dados foram apresentados como média e desvio padrão. O coeficiente de correlação de Pearson foi utilizado para estabelecer a correlação entre os valores de ITB com as variáveis avaliadas no TDBP. Um nível de significância maior ou igual a $5 \%$ foi utilizado para determinar se a associação 
Tabela 1 - Patologias associadas e medicamentos em uso

\begin{tabular}{lcll}
\hline Patologias associadas & (n) & Medicamentos & (n) \\
\hline Hipertensão & 16 & Anticoagulante & 3 \\
Dislipidemia & 4 & Nitrato & 3 \\
Diabetes & 5 & Inibidor da ECA & 7 \\
Angina estável & 2 & Betabloqueador & 5 \\
Tromboangeíte obliterante & 1 & Hemorreológico & 1 \\
Insuficiência cardíaca crônica & 1 & Antitrombótico & 9 \\
Infarto & 1 & Hipolipemiante & 9 \\
Acidente vascular cerebral & 1 & Cilostasol & 6 \\
Tabagismo & 11 & Diurético & 8 \\
Ex-tabagista & 8 & Antagonista canal de cálcio & 5 \\
& & Antiarrítmico & 1 \\
\end{tabular}

entre as medidas foi diferente de zero. O teste $t$ de Student foi utilizado para comparar as variáveis do TDBP no início do sintoma claudicante e durante o sintoma claudicante limitante.

\section{Resultados}

Vinte e nove pacientes foram contatados para participar do estudo, mas apenas 21 foram incluídos. Os outros 8 não compareceram para a realização do teste. Dos 21 participantes, 15 eram homens e 6 mulheres com idade variando de 35 a 80 anos (média 61,95 $\pm 10,21$ anos). A altura média foi de $163,00 \pm 7,00$ centímetros e peso corporal médio foi de 70,16 $\pm 14,68 \mathrm{~kg}$. Todos os pacientes toleraram os procedimentos e não houve complicações ou emergências médicas durante o estudo.

Os pacientes tiveram diagnóstico de DAOP confirmado pela clínica (presença de claudicação intermitente) e através de estudo ultra-sonográfico (Doppler scan). Cinco pacientes apresentaram lesão obstrutiva em um membro e 16 apresentaram em ambos membros inferiores. O tempo médio de diagnóstico de DAOP foi de 54,4 meses, mínimo 12 meses, máximo 216 meses.

Quinze pacientes encontravam-se em programa de reabilitação para doença vascular periférica ou faziam caminhadas programadas. O tempo médio de participação em programa de reabilitação foi de 15,2 meses, variando de 1 a 36 meses. A FC de um paciente durante sintoma limitante não foi registrada por problemas técnicos. Onze pacientes eram tabagistas, 8 ex-tabagistas e 2 pacientes nunca fizeram uso de cigarros. Hipertensão Arterial e Diabetes Mellitus foram as doenças associadas mais encontradas entre os participantes (Tabela 1). As medicações em uso estão listadas na Tabela 1.

Dos 21 pacientes envolvidos no estudo, apenas em um não foi possível registrar o ITB após esforço. $\mathrm{O}$ valor médio de ITB-r foi de $0,66 \pm 0,14$, com intervalo de confiança de $95 \%$ (IC95\%) = 0,38 a 0,94; e o valor médio de ITB-e foi de $0,42 \pm 0,19$, com IC95\% = 0,24 a 0,61. Houve uma redução de $0,24 \pm 0,14$ na média do ITB obtido após o esforço em relação ao ITB de repouso ( $p<0,0005)$. Os valores médios de distância, tempo, velocidade e freqüência cardíaca de início de dor claudicante, bem como de surgimento do sintoma claudicante limitante estão descritos na Tabela 2. Um paciente não relatou sintoma claudicante inicial e, portanto, não foi incluído na estatística correlacional.

A correlação entre o ITB-r com a distância, tempo e velocidade de início de sintoma claudicante e de sintoma claudicante limitante foi pequena e não significante, variando entre 0,19 a 0,25 . O ITB-e não demonstrou correlação importante com nenhuma das 
Tabela 2 - Distância, tempo, velocidade e freqüência cardíaca observados no início do sintoma e durante o sintoma limitante no teste de deslocamento bidirecional progressivo

\begin{tabular}{|c|c|c|c|c|c|c|c|c|}
\hline & \multicolumn{4}{|c|}{ Início sintomas } & \multicolumn{4}{|c|}{ Sintoma limitante } \\
\hline & $\begin{array}{l}\text { Distância } \\
\text { (m) }\end{array}$ & Tempo (s) & $\begin{array}{c}\text { Velocidade } \\
(\mathrm{m} / \mathrm{s})\end{array}$ & FC (bpm) & $\begin{array}{c}\text { Distância } \\
(\mathbf{m})^{*}\end{array}$ & Tempo (s)* & $\begin{array}{c}\text { Velocidade } \\
(\mathrm{m} / \mathrm{s})^{*}\end{array}$ & FC (bpm) \\
\hline $\mathrm{n}$ & 20 & 20 & 20 & 20 & 21 & 21 & 21 & 20 \\
\hline Média & 261,07 & 292,30 & 1,23 & 111,35 & 369,52 & 377,71 & 1,46 & 116,80 \\
\hline DV & 160,63 & 122,61 & 0,34 & 19,39 & 157,97 & 104,60 & 0,29 & 21,15 \\
\hline \multirow[t]{2}{*}{ IC $95 \%$} & 146,65 & 164,20 & 0,69 & 62,55 & 211,48 & 216,17 & 0,83 & 65,61 \\
\hline & 375,49 & 420,40 & 1,77 & 160,15 & 527,57 & 539,26 & 2,08 & 167,99 \\
\hline
\end{tabular}

$\mathrm{DV}=$ desvio padrão; $\mathrm{FC}=$ freqüência cardíaca obtida em 20 pacientes; IC95\% = intervalo de confiança de 95\%; $\mathrm{n}=$ número de indivíduos testados.

$* \mathrm{p}<0,05$ (início sintomas versus sintoma limitante).

variáveis obtidas, tanto no início do sintoma claudicante quanto durante o sintoma claudicante limitante (variando de $-0,01$ a 0,12 ).

As variáveis funcionais apresentaram correlações mais significativas entre si quando o início de surgimento do sintoma claudicante foi comparado com o sintoma claudicante limitante. As correlações entre distância, tempo e velocidade até o início do sintoma claudicante com a distância, tempo e velocidade totais alcançada até o sintoma limitante foram de 0,92 ( $\mathrm{p}<0,0005), 0,91(\mathrm{p}<0,0005)$, e $0,89(\mathrm{p}<0,0005)$, respectivamente.

Não houve diferença estatisticamente significativa entre a FC registrada no início do surgimento do sintoma claudicante e durante a FC do sintoma limitante (Tabela 2). A FC não apresentou correlação significativa com a distância, tempo e velocidade durante o surgimento do sintoma claudicante. Já a FC durante o sintoma limitante apresentou correlação de $0,55,0,55$ e $0,49$ ( $\mathrm{p}<0,0005)$ com distância, tempo e velocidade durante claudicação limitante, respectivamente.

\section{Discussão}

O objetivo principal deste estudo foi determinar o grau de associação do ITB-e com um novo teste de deambulação, o TDBP. A escolha pelo ITB após a realização da atividade física deveu-se ao fato de que a literatura apresenta correlações variáveis do ITB-r com performance ${ }^{1}$. Outros estudos sugerem que o ITB-e possa apresentar níveis de correlação um pouco melhores, embora seu uso ainda não tenha sido investigado do ponto de vista da associação com capacidade funcional e variáveis hemodinâmicas ${ }^{3,5}$. A escolha pelo TDBP foi devido a sua padronização, menor influência quanto ao encorajamento do paciente, vantagens em se desenvolver caminhada no solo a velocidades incrementais, além de apresentar maior estabilidade nas medidas de reprodutibilidade ${ }^{13}$.

McDermontt et al. sugeriram que o ITB medido após a realização da atividade física poderia, potencialmente, apresentar níveis de correlação melhores com performance que o ITB-r, já que o portador de DAOP pode apresentar uma queda de mais de $25 \%$ no ITB-e $e^{5,14}$. No presente estudo houve uma redução de cerca de $36 \%$ no índice obtido após o esforço, mas não se observou correlação substancial deste índice com as medidas de tempo, velocidade ou distância de início de sintoma ou de sintoma limitante. A correlação entre o ITB de repouso e as variáveis funcionais citadas foram pequenas e não significantes.

Stein et al. demonstram que a associação entre ITB e função foi forte quando se calculou o ITB utilizando-se a média dos valores entre a artéria dorsal do pé e tibial posterior $^{2}$. No presente estudo, contudo, optou-se por utilizar o valor mais baixo de pressão sistólica aferida no membro inferior, por se julgar que a maior obstrução tenha maior impacto sobre a função do membro. Entretanto, estudos prévios utilizavam a maior pressão 
do braço e da perna para o cálculo do $\mathrm{ITB}^{1,15}$. Portanto, é possível que as variações no registro do ITB expliquem, em parte, a variabilidade dos valores de correlação com medidas funcionais.

Outra explicação possível para a correlação precária entre ITB e as variáveis funcionais pode se dever ao fato de que todos os pacientes estavam em uso de medicações que podem alterar o fluxo sangüíneo e irrigação do membro afetado. Houve pacientes cuja medicação poderia facilitar o fluxo (cilostazol, inibidores da ECA, antagonistas de canal de cálcio, hemorreológicos) ou comprometê-lo (betabloqueador).

Outro fator que possa contribuir para explicar a baixa ou falta de correlação entre os ITB de repouso e pós-esforço com o TDBP se deve ao nível de atividade física dos participantes. Tem sido demonstrado que o paciente treinado tem melhores mecanismos de captação e utilização periférica de oxigênio, sendo tais fatores os que, possivelmente, melhor explicam a atenuação sintomatológica do paciente claudicante em testes funcionais, a despeito da não alteração no fluxo sangüíneo estimada pelo ITB ${ }^{1}$. No presente estudo, $76 \%$ dos pacientes faziam parte de um programa de atividade física e, portanto, já poderiam exibir adaptações periféricas que influenciassem a associação entre fluxo sangüíneo e performance. Gardner et al. demonstraram que o aumento de fluxo avaliado pela resposta hiperêmica (27\%) e no ITB (1\%) foram bem menores que a melhora na distância caminhada até o início de dor (133\%) e até o surgimento de dor claudicante máxima (77\%) após um programa de treinamento ${ }^{3}$.

O comportamento cronotrópico também suporta a hipótese de que alguma adaptação periférica esteja presente. Primeiro, não houve diferença estatisticamente significativa entre as freqüências cardíacas de início de sintoma e de sintoma limitante, a despeito das diferenças estatisticamente significativas nas variáveis de performance no teste. Portanto, isso sugere que o desempenho durante o teste possa estar mais relacionado à capacidade de utilização de oxigênio do que aos mecanismos de distribuição. Além disso, não houve correlação entre a FC do início do sintoma com qualquer variável no teste de caminhada, enquanto que a variância compartilhada $\left(\mathrm{r}^{2}\right)$ entre $\mathrm{FC}$ e as variáveis durante o sintoma limitante foi de apenas 30\%.

Se por um lado não se pôde demonstrar correlação entre os índices de comprometimento de fluxo e performance com o TDBP nessa população, o TDBP demonstrou ser consistente quanto à avaliação dos sintomas clínicos. O tempo, distância e velocidade de início de surgimento de sintoma apresentaram correlações fortes e significativas com as referidas variáveis obtidas durante o surgimento do sintoma limitante (coeficiente de correlação variando de 0,89 a 0,92, p < 0,05). Desta forma, o TDBP apresenta-se como um instrumento viável para avaliação de sintomas em pacientes com DAOP.

\section{Limitações do estudo}

$\mathrm{O}$ reduzido número de participantes, bem como o uso de medicações e falta de uniformidade em relação ao nível de atividade física são questões que devem ser consideradas em estudos futuros, já que podem ter interferido nos resultados. A forma mais adequada de registro do ITB também necessita ser investigada.

\section{Conclusões}

A redução ulterior do ITB observada após o esforço neste estudo corrobora o comprometimento do fluxo no paciente com DAOP. Entretanto, a baixa ou falta de correlação entre o ITB-r e o ITB-e com a performance no TDBP sugere que o paciente claudicante é capaz de realizar o teste de caminhada às custas, em princípio, de um mecanismo de captação de oxigênio eficaz. O TDBP demonstrou-se consistente ao avaliar tempo, velocidade e distância percorrida durante o início de sintoma e sintoma claudicante limitante. Portanto, esse teste de caminhada progressiva no solo se apresenta como uma alternativa para uma avaliação consistente e prática do paciente na clínica angiológica. Os resultados desse estudo sugerem que a avaliação do grau de comprometimento paciente claudicante não deve prescindir de uma avaliação funcional.

\section{Referências}

1. McDermott MM, Greenland P, Lui K, et al. The ankle brachial index is associated with leg function and physical activity: the walking and leg circulation study. Ann Intern Med. 2002;136:873-83. 
2. Stein R, Hriljac I, Halperin JL, Gustavson SM, Teodorescu V, Olin JW. Limitation of the resting ankle brachial index in symptomatic patients with peripheral arterial disease. Vasc Med. 2006;11:29-33.

3. Feringa $\mathrm{HH}$, Bax JJ, van Waning VH, et al. The long-term prognostic value of the resting and postexercise ankle brachial index. Arch Intern Med. 2006;166:529-35.

4. Wolosker N, Rosoky RA, Nakano L, Basyches M, PuechLeão P. Predictive value of the ankle-Brachial index in the evaluation of intermittent claudication. Rev Hosp Clin Fac Med São Paulo. 2000;55:61-4.

5. McDermott MM, Liu K, Guralnik JM, et al. The ankle brachial index independently predicts walking velocity and walking endurance in peripheral arterial disease. $\mathrm{J}$ Am Geriatr Soc. 1998;46:1355-62.

6. Coughlin PA, Kent PJ, Turton EP, et al. A new device for measurement of disease severity in patients with intermittent claudication. Eur J Vasc Endovasc Surg. 2001;22:516-22.

7. Gardner AW, Montgomery PS, Flinn WR, Katzel LI. The effect of exercise intensity on the response to exercise rehabilitation in patients with intermittent claudication. J Vas Surg. 2005;42:702-9.

8. Cachovan M, Rogatti W, Woltering F, et al. Randomized reliability study evaluating constant-load and graded-exercise treadmill test for intermittent claudication. Angiology. 1999;50:193-200.

9. Gardner AW, Skinner JS, Cantwell BW, Smith LK. Progressive vs single-stage treadmill tests for evaluation of claudication. Med Sci Sports Exerc. 1991;23:402-8.
10. Montgomery PS, Gardner AW. The clinical utility of a sixminute walk test in peripheral arterial occlusive disease patients. J Am Geriatr Soc. 1998;46:706-11.

11. Zwierska I, Nawaz S, Walker RD, Wood RF, Pockley AG, Saxton JM. Treadmill versus Shuttle Walk Tests of walking ability in intermittent claudication. Med Sci Sports Exerc. 2004;36:1835-40.

12. Green DJ, Watts K, Rankin S, Wong P, O'Driscoll JG. A comparison of the shuttle and 6 minute walking tests with measured peak oxygen consumption in patients with heart failure. $\mathrm{J}$ Sci Med Sport. 2001;4:292-300.

13. da Cunha-Filho IT, Pereira DA, de Carvalho AM, et al. The Reliability of Walking Tests in people with claudication. Am J Phys Med Rehabil. 2007;86:574-82.

14. McDermott MM, Liu K, Greenland P, et al. Functional decline in peripheral arterial disease. Associations with the ankle brachial index and leg symptoms. JAMA. 2004;292:45361.

15. Gardner AW, Skinner JS, Cantwell BW, Smith LK. Prediction of claudication pain from clinical measurements obtained at rest. Med Sci Sports Exerc. 1992;24:163-70.

Correspondência:

Inácio Cunha-Filho

Rua Ernani Agrícola 50/302

CEP 30455-760 - Belo Horizonte, MG

Tel.: (31) 3378.8758

Fax: (31) 2108.7856

E-mail: inacutex@terra.com.br 